\title{
Acknowledgment to MIR Board Members and Ad-hoc Reviewers
}

We would like to thank all members of the Editorial Board and the Editorial Review Board for their valuable inputs they have provided in the year 2012 for the further advancement of Management International Review. Furthermore, we would like to thank the following scholars who supported Management International Review in the last year 2012 as ad-hoc reviewers:

Sönke Albers, Nicholas Athanassiou, Ellie Banalieva, Florian Becker-Ritterspach, Elena Beleska-Spasova, Nicola Berg, Arjun Bhardwaj, Torsten Biemann, Julian Birkinshaw, Christian Blecher, Timothy P. Blumentritt, Jean J. Boddewyn, Keith Brouthers, Murali Chari, Tain-Jy Chen, Benoit Chevalier-Roignant, John Child, Jeremy Clegg, Joseph Clougherty, Nicole E. Coviello, Alvaro Cuervo-Cazurra, Michael R. Czinkota, Mehmet Demirbag, Gregory G. Dess, Christoph Dörrenbächer, Rian Drogendijk, Anna Dubiel, Till Dunemann, Stefan Eckert, Matthias Ehrgott, Martin Eisend, Prescott Ensign, Jürgen Ernstberger, Dorothee Feils, Mark Fenton, Marion Festing, Karin M. Fladmoe-Lindquist, Kathy Fogel, Manfred Fuchs, José Ignacio Galan, Katja Gelbrich, Jane K. Giaccobe-Miller, Keith Wilson Glaister, Anthony Goerzen, Gary Gregory, Christoph Grimpe, Robert Grosse, Siegfried Gudergan, Mohamed Gulamhussen, Amjad Hadjikhani, Lars Håkanson, Niron Hashai, Arno Haslberger, Louis Hébert, Martin Hemmert, Michael Hitt, Martin Högl, Ulf Holm, Chin-Chun Hsu, Jae Jung, Rüdiger Kabst, Lutz Kaufmann, Kwanghyun (Harry) Kim, Dodo zu Knyphausen-Aufseß, Markus Kreutzer, Torsten Kühlmann, Lee Li, Siqi Li, Ulrich Lichtenthaler, Peter W. Liesch, Howard Lin, Xiaming Liu, Cristina López-Duarte, Sarianna Lundan, Randi Lunnan, Mary M. Maloney, Stephan Manning, Sara Melén, Klaus Meyer, Carla Millar, Dana Minbaeva, Fiona Moore, Susan Mudambi, Phillip Nell, William Newburry, Stephen Nicholas, Niels G. Noorderhaven, Jana Oehmichen, Thomas Osegowitsch, Marina Papanastassiou, Ronaldo Parente, Bent Petersen, Heike Proff, Markus Pudelko, Gongming Qian, Roberto Ragozzino, B. Sebastian Reiche, Thomas W. Roehl, Jean-Louis F. Schaan, Andreas Schotter, Arnold Schuh, Carsten Schultz, Joanna ScottKennel, S. Prakash Sethi, Joshua D. Shackman, Deo Sharma, Rudolf R. Sinkovics, Minna Söderqvist, Carl Arthur Solberg, Colette Southam, Pauline Stanton, Thomas Steger, Bruce W. Stening, Michael Stephan, Barbara Stöttinger, Roger Strange, Sully Taylor, Georg Trautnitz, Jan-Erik Vahlne, Achim Walter, Sascha Walter, Catherine Welch, Christopher Williams, Shubin Wu, Yanni Yan, Eden Yin, Ivo Zander, Hongxin Zhao

Michael-Jörg Oesterle (Co-Editor-in-Chief)
Joachim Wolf (Co-Editor-in-Chief)

(C) Springer Fachmedien Wiesbaden 2013 